\title{
Examining recognition criterion rigidity during testing using a biased-feedback technique: Evidence for adaptive criterion learning
}

\author{
SANGHOON HAN \\ Duke University, Durham, North Carolina \\ AND \\ IAN G. DOBBINS \\ Washington University, St. Louis, Missouri
}

\begin{abstract}
Recognition models often assume that subjects use specific evidence values (decision criteria) to adaptively parse continuous memory evidence into response categories (e.g., "old," "new"). Although explicit pretest instructions influence criterion placement, these criteria appear extremely resistant to change once testing begins. We tested criterion sensitivity to local feedback using a novel biased-feedback technique designed to tacitly encourage certain errors by indicating they are the correct choices. Experiment 1 demonstrated that fully correct feedback had little effect on criterion placement, whereas biased feedback during Experiments 2 and 3 yielded prominent, durable, and adaptive criterion shifts, with observers reporting that they were unaware of the manipulation in Experiment 3. These data suggest that recognition criteria can be easily modified during testing through a form of feedback learning that operates independently of stimulus characteristics and observers' awareness of the nature of the manipulation. This mechanism may be fundamentally different from criterion shifts following explicit instructions and warnings, or shifts linked to manipulations of stimulus characteristics combined with feedback highlighting those manipulations.
\end{abstract}

A common way of characterizing recognition decisions is as a unidimensional signal detection process. In its simplest form, this assumes that performance is governed by a scalar indication of the amount of mnemonic evidence (signal), and a value or set of values that is used to parse that evidence into discrete response categories (decision criterion/criteria) (Macmillan \& Creelman, 1991). In the most common model, researchers assume two normal evidence distributions - one for targets and one for luresoffset by a distance representing the discriminability of the item types. For simple old/new classification, only a single criterion is used to divide the continuum. In contrast, when asked to make confidence ratings, observers are assumed to employ multiple criterion values to parse the continuum into the required number of confidence ratings. When the old and new item endorsement rates are cumulated from most to least confident, a characteristic curve known as the receiver operating characteristic (ROC) is traced out (Figure 1). The ROC function is important because it specifies all the old and new item response rates that are permissible for a given level of accuracy $\left(d^{\prime}\right)$ as a result of differently placed decision criteria.

Critically, although observers are assumed to have little strategic control over the distributions of evidence that are available during recognition testing, the placement of deci- sion criteria is often assumed to be under a high level of observer control, with observers being capable of shifting criterion positions in order to favor particular outcomes. For example, if subjects are rewarded for detecting old items, yet face no punishment for incorrectly endorsing new items, then it would be advantageous to rapidly shift the old/new criterion to a more lax position by increasing the correct old rate and, hence, increasing the reward. However, despite its intuitive appeal and clear adaptive advantage, the extant evidence is highly mixed with regard to the ability of subjects to adaptively reposition recognition decision criteria. We briefly review this evidence below, beginning with the one manipulation that does appear to reliably invoke adaptive memory criterion positioning, namely, explicit pretest instructions or warnings.

Several instruction methods yield adaptive memory criterion shifts when presented prior to testing. These include instructing subjects to favor either high-confidence "old" or "new" responses (Azimian-Faridani \& Wilding, 2006), providing them with either veridical or misleading information about upcoming target-lure ratios (Hirshman \& Henzler, 1998; Rotello, Macmillan, Reeder, \& Wong, 2005; Strack \& Förster, 1995), or informing them about differences in relative payoffs for certain outcomes (Van Zandt, 2000). In all cases, it is important to note that the

S.Han, sanghoon.han@duke.edu 

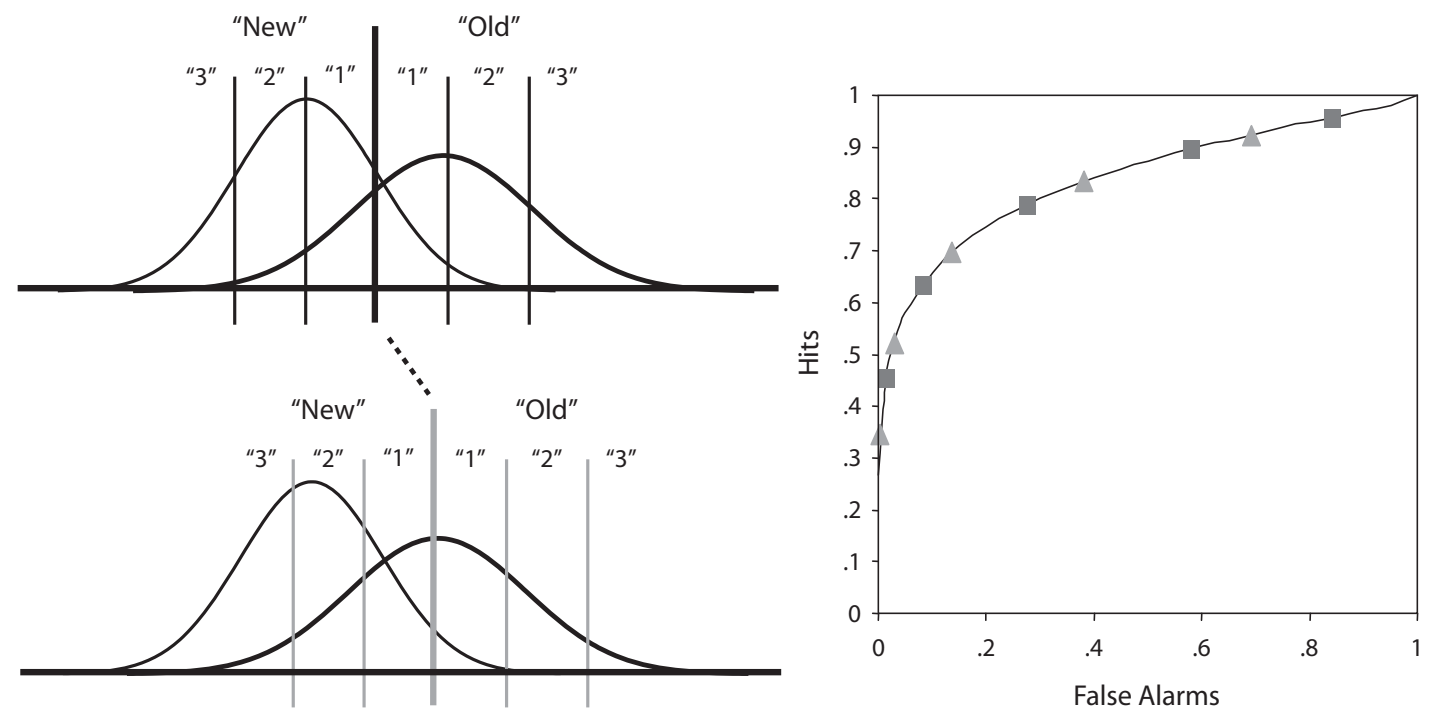

Figure 1. Decision criteria and the receiver operating characteristic (ROC) of the unequal variance signal detection model. The left-hand panels illustrate the difference between strict and lax criterion placement in an unequal variance signal detection model where the variance of the old item distribution is greater than that of the new item distribution. When one cumulates the response proportions starting with the most confident ("yes") responses, the ROC is traced out in probability space (shown in the right panel). The example ROC demonstrates a typical asymmetry about the negative diagonal with the left-hand portion of the curve pushed up along the $y$-axis. The relative positioning of the criteria determines where the points fall on the ROC. Whereas the lax criterion points are shown as dark gray rectangles, the shift toward strict responding is shown by the gray triangles. Importantly, the change in criteria simply shifts the points along the same surface, and this reflects the assumption that the criteria can be manipulated independently of the memory evidence.

shift reflects an adjustment that occurs prior to testing as a function of explicit instruction, and that it reflects subjects' explicit understanding of test list characteristics or contingencies that make one response option relatively more desirable or profitable than another. However, the efficacy of explicit instructions in inducing criterion shifts stands in marked contrast to the insensitivity of memory decision criteria to changes in testing conditions that should also encourage adaptive criterion shifts. For example, in a series of early studies, Wallace and colleagues found similar hit rates regardless of whether or not the test lists actually contained lures (Wallace, 1982; Wallace, Sawyer, \& Robertson, 1978). This is striking from a signal detection perspective since one might predict that when lures are entirely absent, observers would capitalize on the perceived high proportion of targets and accordingly adjust the criterion downward in order to maximize success rates. Somewhat similarly, Estes and Maddox (1995) found that the word-recognition criterion appeared to be insensitive to prominent changes in average target density during continuous recognition testing, even when explicit feedback was used to inform performance.

More recently, Verde and Rotello (2007) presented observers with test lists that seamlessly abutted two blocks, each containing either strongly or weakly encoded targets intermixed with novel lures. If subjects spontaneously adjust the recognition decision criterion, then they should adjust the criterion either upward or downward, depending on the average strength of the probes in the two test halves. For example, when transitioning from a strong target block to a weak target block, subjects should adjust the criterion downward because signals exceeding their old/new criterion would suddenly become increasingly rare, suggesting that they were being too stringent (see Glanzer \& Adams, 1990). Nonetheless, even though there were prominent changes in the hit rates between the adjacent blocks, the false alarm rates remained fixed, suggesting that observers' criteria were insensitive to persistent changes in the average strength of the items during testing. Similar extreme forms of criterion rigidity have been observed when different strength levels were associated with different categories of items at test, with subjects failing to adopt different criteria for weak and strong categories of items (see Morrell, Gaitan, \& Wixted, 2002; Stretch \& Wixted, 1998).

Taken as a whole, the studies above suggest that once testing begins, subjects do not use systematic changes in average strength or target density to adaptively shift their criterion. We refer to these systematic manipulations as global in that they relate to a large portion of the test list or an entire class of items within the list. Whether subjects are unaware of these manipulations, simply do not understand how they should adjust their responses because of them, or are unwilling to expend the effort to capitalize upon them, remains unclear. However, the studies above suggest strong limits on subjects' abilities to use global test list regularities as cues to more effectively reposition recognition decision criteria. 
Despite these findings, which suggest extremely rigid criterion placement during the course of testing, there are also studies that indicate that subjects can adjust the criterion position on a trial-by-trial basis, as a function of the semantic characteristics of individual retrieval probes. For example, subjects appear to adopt a stringent criterion for items that are personally distinctive and known (e.g., a picture of one's favorite coffee shop) versus those that may be perceptually similar but unknown (e.g., an unknown coffee shop) (Dobbins \& Kroll, 2005). Such shifts are in accord with the subjective memory heuristic postulated by Brown, Lewis, and Monk (1977) that assumes that memory decisions are informed by subjective expectancies about the memorability of encounters with personally unique and relevant stimuli (Benjamin, 2003; see also Benjamin \& Bawa, 2004). Importantly, in all these cases that indicate trial-by-trial criterion adjustment, the adjustments are made in response to the differing semantic characteristics of the probes across the trials.

In the summary of the work on verbal recognition criteria outlined above, it appears that criterion shifts have been readily documented only under two circumstances. First, when the semantic characteristics of each individual probe can be clearly used to estimate memorability, then subjects appear capable of adjusting the criterion strategically. Second, if subjects are provided with explicit instructions that make the preferred response strategy clear, then criterion shifts are also obtained. In both cases, subjects are likely to be able to explicitly state why they are responding cautiously or liberally on the basis of known characteristics of the test list construction or of semantic features of the individual probes themselves. Conversely, however, the data also suggest that subjects are unable to capitalize upon either global changes in probe strength or density (or response rate changes associated with these manipulations) in order to reposition their criteria adaptively.

In the present study, we assume that although subjects are insensitive to global manipulations of probe strength or target density, recognition decisions should nonetheless be highly sensitive to local, trial-specific cues that inform current criterion utility. The method we used to test this assumption is a subtle and novel feedback manipulation. Previous research suggests that subjects can effectively use feedback to guide and monitor performance in nonrecognition memory domains (Bohil \& Maddox, 2003; Elliott, Frith, \& Dolan, 1997; Maddox \& Bohil, 2001; Papo, Baudonnière, Hugueville, \& Caverni, 2003; Pashler, Cepeda, Wixted, \& Rohrer, 2005). Additionally, psychophysical studies have proposed learning models in which subjects adjust criteria in response to feedback after errors occur (Dorfman \& Biderman, 1971; Kubovy \& Healy, 1977; Thomas, 1973). However, despite the prevalence of feedback manipulations in nonmemory domains, the use of feedback information to attempt to influence memory decision criteria is surprisingly rare, and in fact we know of only three reports of such attempts (Estes \& Maddox, 1995; Rhodes \& Jacoby, 2007; Verde \& Rotello, 2007).

Verde and Rotello (2007) were able to induce a criterion change by providing subjects with correct feedback on every trial—stopping intermittently and giving subjects quartile performance summaries - and by globally reducing the average strength of targets in the second half of the test relative to the first half (Experiment 4). Although these researchers were able to induce a more liberal criterion, it is not clear which aspect of the design was critical. The adjustment could have required the prominent change in average target strength; the trial-by-trial feedback may have been the necessary component; or the quartile performance summaries may have functioned in a manner similar to providing explicit instructions to be wary of certain response types. In a continuous recognition paradigm (i.e., Shepard, 1967), Estes and Maddox (1995) were also able to induce a criterion shift during digit and letter-string recognition memory by using feedback coupled with changing target probability. However, this manipulation was ineffective for verbal recognition, which was the focus of our investigation. Finally, Rhodes and Jacoby (2007) demonstrated a criterion shift by pairing screen locations with different target probabilities and providing feedback. Their data suggested that subjects adopted different criteria for the locations, provided that they explicitly realized that the regions were associated with different ratios of targets to lures. This manipulation required a prominent manipulation of the distributions of memoranda, however; in this case, this entailed the crossing of different target densities with different locations, coupled with extensive feedback.

It is important to note that in the two cases where feedback has been effective in shifting word recognition decision criteria, the shift only occurred in conjunction with a prominent manipulation of average strength or target probability coupled with extensive feedback procedures. On the basis of the nature of the feedback provided in Verde and Rotello (2007) and the findings of Rhodes and Jacoby (2007), it is likely that the feedback in these paradigms functioned similarly to an explicit instruction condition clearly warning subjects to strategically favor or avoid certain classifications because of global changes in the strength or probabilities of memory targets. For example, in Verde and Rotello, not only was feedback provided on every trial, but also the test was paused and performance summaries were given for every quartile of the test that showed the total number of correct and incorrect responses for that test segment. Somewhat similarly, in Rhodes and Jacoby, feedback was given on every trial, and a running total of correct responses was shown to subjects throughout. These authors also found that additional manipulations that made the different target probabilities even more salient - such as using different keys for the different presentation quadrants - strengthened the effect. These effects, combined with postexperiment questionnaires, led Rhodes and Jacoby to conclude that the effect heavily depended upon conscious awareness of how the memoranda were being spatially manipulated.

Critically then, not a single study in the memory literature demonstrates that subjects are able to adaptively adjust the decision criterion during testing unless there are also prominent manipulations of the memoranda coupled with feedback conditions or stimulus properties that likely make subjects explicitly aware of appropriate response 
strategies, given these manipulations. If such conditions were absolutely necessary, this would represent a critical limitation of memory criterion setting and stand in stark contrast to the assumption of a highly flexible criterion that is fully independent of the distribution of evidence (Macmillan \& Creelman, 1991). The primary goal of the present study was to determine whether, in the absence of any alteration of the memoranda, subjects could nonetheless adaptively shift the decision criterion. Furthermore, we were interested if the shift could be induced in the absence of awareness of the manipulation used to induce it. If so, this would stand in marked contrast to prior successful instruction and feedback manipulations and might reflect a type of learning not previously considered relevant during explicit recognition decisions.

\section{Adaptive Criterion Learning}

To attempt to induce criterion shifts, we used a subtle biased-feedback technique that selectively misinformed subjects during errors of commission (false alarms) or errors of omission (misses), whereas correct responses were always indicated as such. By falsely informing subjects about certain types of errors, we expected them to learn to favor the response type that would lead to a positive outcome more often, or, analogously, to learn to avoid the response that was associated with negative outcomes more often. Furthermore, because the manipulation was restricted to errors, we reasoned it would be largely transparent to the subjects. This approach has parallels with studies examining the matching law (e.g., Herrnstein, 1970) and theories suggesting a link between reinforcement learning theory and memory performance (e.g., White \& Wixted, 1999). The matching law refers to the reliable tendency of animals to distribute responses probabilistically between response options as a function of their prior probabilities of reinforcement. Importantly, a wide range of animals do this, including species that are unlikely to be capable of forming explicit response strategies. Importantly, any systematic learned favoring of one response type over another as a function of reinforcement learning would appear as a measurable bias in the animals' memory choices (White \& Wixted, 1999), although it might reflect the joint operation of two learning mechanisms: one that resulted in the laying down of the episodic trace, and one that governed the learning of response preferences. Finally, as we elaborate in the General Discussion section, if the false feedback technique were effective, it would also share links with the response changes observed during probabilistic classification learning (Knowlton, Squire, \& Gluck, 1994), a form of learning that requires neither changes in the stimulus materials nor explicit awareness of the test list contingencies.

\section{EXPERIMENT 1 \\ Unbiased Feedback Versus Nonfeedback (Old/New Recognition)}

Before we assessed whether the biased-feedback manipulation would induce criterion shifts, Experiment 1 was conducted to determine whether providing unbiased feedback during recognition memory judgments would, in and of itself, appreciably affect accuracy or criterion placement. The presence or absence of feedback was manipulated within subjects in order to maximize sensitivity. As we note below, finding a prominent influence of feedback on accuracy estimates would complicate matters because the interpretation of criterion shifts is potentially complicated when confounded with concomitant changes in accuracy (Pastore, Crawley, Berens, \& Skelly, 2003; Wixted \& Stretch, 2000).

\section{Method}

Subjects

Sixteen Duke University undergraduate students participated, in return for course credits. Informed consent was obtained, as required by the human subjects review committee of the university.

\section{Materials and Procedure}

A total of 480 nouns was drawn randomly for each subject from a pool of 1,216 words total. From the list, four lists of 120 items (60 old, 60 new items for each cycle) were constructed for use in four study/test cycles. The items in the pool had on average 7.09 letters, and 2.34 syllables, with a Kučera-Francis corpus frequency of 8.85 . The subjects were tested individually, and computers were used for the presentation of items and the collection of responses. The order of feedback blocks and nonfeedback blocks was randomized.

Prior to study, all subjects were instructed that they would be required to study a list of items while counting the syllables of each word and that they would be immediately tested on their memory for the items. Subjects were not forewarned that feedback would be present on half the blocks. After 10 practice trials, which were used to familiarize subjects with the key assignments, subjects pressed a key to begin the study proper. For encoding, subjects were instructed to report the number of syllables for each serially presented word. Each word appeared with the task cue "Counting syllables $1 / 2 / 3 /$ more than 4 " underneath, and subjects were given a limited amount of time $(2 \mathrm{sec})$ to perform the task. If no response was entered within $2 \mathrm{sec}$, a warning message that asked for a quicker response was presented, along with a beep. There were 60 words in each study phase. In each subsequent memory test, the studied items were intermixed with 60 new items and presented serially for yesno recognition judgments ("Is this OLD or NEW? $1=$ OLD $2=$ NEW") and confidence ratings ("Confidence? Unsure = $123=$ Certain") using preassigned keys on the keyboard. Responses were self-paced. There were four study/test blocks.

Feedback manipulation. Two of the four test blocks were accompanied by feedback that noted the accuracy of each memory response, with the order determined randomly for each subject. Following the subject's confidence report, feedback was presented that indicated whether the response was correct ("That is CORRECT") or incorrect ("That is INCORRECT"). These prompts were located directly beneath the test probe; they were bright orange and accompanied by a beep for incorrect responses. Feedback for correct responses was in the same font color as the probes and did not have an accompanying sound.

\section{Results and Discussion}

\section{Accuracy $\left(\mathrm{A}_{\mathrm{z}}\right)$ and Decision Criteria $\left(\mathrm{c}_{\mathrm{a}}\right)$}

The analyses employed the detection theoretic estimates of accuracy and decision bias $\mathrm{A}_{\mathrm{z}}$ and $\mathrm{c}_{\mathrm{a}} \cdot{ }^{1} \mathrm{~A}$ contrast of $\mathrm{A}_{\mathrm{z}}$ across the feedback $\left(\mathrm{A}_{\mathrm{z}}=.90\right)$ and no-feedback $\left(\mathrm{A}_{\mathrm{z}}=\right.$ .90) conditions suggested that overall accuracy was unaffected by feedback $[t(15)=0.02, p>.98]$. Similarly, the estimate of response bias, $c_{a}$, did not significantly differ across the feedback $\left(c_{a}=.16\right)$ and no-feedback $\left(c_{a}=.10\right)$ 
conditions $[t(15)=0.90, p>.37]$. The positive values of $\mathrm{c}_{\mathrm{a}}$ suggested that subjects were, on average, slightly conservative, whereas negative values of $c_{a}$ indicated liberal responding and a greater willingness to say "old" (Macmillan \& Creelman, 1991).

Although the estimates of accuracy and bias estimates did not differ, the collection of confidence reports allowed us to visually inspect the ROCs for each condition. Figure 2 shows the aggregate ROCs for the two feedback conditions and indicates that they fell along the same curve, and that unbiased feedback during recognition memory had little effect on the information upon which subjects based their responses. The data also demonstrate the asymmetry commonly observed in item recognition studies, in that the left portion of the ROC appears "pushed up" along the $y$ axis and the plot is not symmetric about the negative diagonal (Ratcliff, Sheu, \& Gronlund, 1992). This shape is consistent with a one-dimensional signal detection account (in which the old item evidence values are more variable than the new item values; e.g., Figure 1) and is similar to old $/$ new variance ratios observed in other studies $\left(1 / \sigma_{\text {old }}=.70\right)$.

Overall, the results suggest that neither recognition accuracy nor criterion location differed significantly as a function of the presence or absence of performance feedback. This is informative, since there are at least two reasons why one might instead predict that feedback would improve performance. First, subjects might benefit from feedback if it were useful in determining which types of probe information were or were not diagnostic of prior study. That is, if subjects sometimes believed that tangential probe characteristics, such as word frequency or concreteness, were directly diagnostic of study status, feedback might help to disavow these erroneous beliefs and therefore improve performance. Alternatively, one

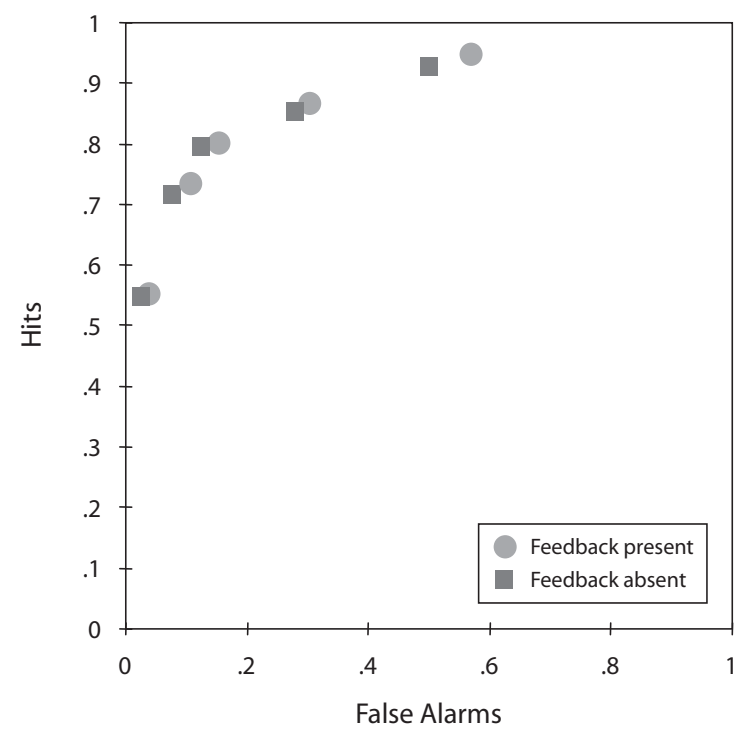

Figure 2. Recognition memory ROCs from Experiment 1 for feedback-present (circle) and feedback-absent (box) conditions. might suppose that, in the absence of feedback, subjects may "hunt" for an appropriate old/new criterion location across trials. If the old/new decision criterion varied in its position from trial to trial, feedback could help minimize this criterion variance by informing subjects more quickly and directly about the appropriateness of a chosen location. This would, in turn, elevate performance because trial-to-trial changes in the criterion location reduce the observed accuracy of the observer (Carterette, Friedman, \& Wyman, 1966; Friedman, Carterette, \& Nakatani, 1968; Treisman \& Williams, 1984). Although plausible, neither of these hypotheses was supported by the results.

The null effect of veridical feedback on the accuracy data in Experiment 1 indicates that any criterion changes observed in the feedback manipulations we used in the following experiments will likely be interpretable. Had the feedback resulted in prominent differences in accuracy, its utility for examining criterion effects would have been potentially reduced, since there is some degree of uncertainty as to what constitutes the appropriate criterion measure when accuracy also changes (see Pastore et al., 2003).

\section{EXPERIMENT 2 Biased-Feedback Procedure (Old/New Recognition: Two-Step Classification)}

To examine the potential for feedback to induce criterion shifts that are independent of stimulus characteristics and explicit instructions, in Experiment 2 decision/outcome contingencies were subtly manipulated in an attempt to encourage either misses or false alarms in the context of feedback that was otherwise generally correct. For example, if a subject received false feedback that indicated that he or she was in fact correct during miss trials, then adopting an even a stricter criterion was potentially desirable, since further movement in this direction would yield an even greater ratio of positive - relative to negativeoutcomes, if the remaining feedback for the three other response types was accurate. In contrast, movement in a more lax direction would be discouraged by the presence of accurate feedback that would correctly identify false alarms. The remaining two correct response types - hits and correct rejections - are always correctly identified during the biased-feedback procedure. Restricting the biasing feedback to errors also had the added advantage of helping to ensure that the manipulation remained transparent to observers, since they do not receive feedback that is inconsistent with their most confident responses, which, overwhelmingly, are confined to correct responses. Instead, because the manipulation was confined to errors, which tend to be of low confidence, subjects had little basis to view the feedback as suspect or unreliable. Overall then, the prediction motivating the biased-feedback procedure was that subjects would increasingly learn to favor the response option that led to a greater net increase in positive outcomes (or net decrease in negative outcomes) and to do so without awareness that the feedback was purposely skewed. The same logic applied to applying false feedback to false alarms, which should have induced a lax drift if subjects learned to favor the response 
type associated with greater net positive outcomes. To our knowledge, this is the first time that this type of feedback has been used to try to induce biased responding during recognition, or for that matter during any cognitive or perceptual discrimination.

The potentially tacit nature of the biased-feedback manipulation stands in contrast to the use of explicit instructions that are meant to influence criterion positioning, and we consider the potential for these two approaches to rely on different mechanisms in the General Discussion. Finally, because the actual biased-feedback manipulation only occurs on a minority of trials (errors of one or the other type), any demonstration of a shift would suggest that subjects are quite sensitive to changes in the response contingencies. This would offer a clear contrast to the insensitivity of recognition criteria to the global list manipulations discussed in the introduction.

\section{Method}

\section{Subjects}

Thirty Duke University undergraduates participated in return for partial course credit. Informed consent was obtained, as required by the human subjects review committee of Duke University. The subjects were randomly assigned to two groups in which the nature of the feedback was differently adjusted across separate tests. Subjects who demonstrated abnormally low performance $\left(d^{\prime}<0.5\right)$ were removed and replaced. One subject met this criterion and was removed from Experiment 2.

\section{Materials and Procedure}

Using the same item pool as that in Experiment 1, a total of 600 nouns were randomly drawn for each subject. From this total, three lists of 200 items (100 studied and 100 lure items) were constructed for use in three sequential study/test cycles. The study period was identical for all cycles, and for both groups of observers. What differed was the nature of the biased feedback delivered during each test. The assignment of items to targets or lures was randomized, and the order of test items was also randomized for each subject and cycle. Subjects were not warned of the feedback prior to the beginning of Test 1 . The orienting task during study and the response requirements during test were identical to those in Experiment 1. Again, reporting was self-paced.

Feedback manipulation. After reporting the old/new judgment and confidence, accuracy feedback was immediately presented. The nature of the feedback across the three tests was manipulated between groups. For half the subjects, the first test selectively encouraged lax responding by giving false positive feedback- "That is CORRECT" for the incorrect old classification of new items (false alarms). All other response types (i.e., hits, misses, and correct rejections) were correctly identified by the feedback. We refer to this as the lax (L) feedback condition. In the second block, this group received feedback that was correct for all response types (N, neutral condition). Finally, in the third and final test, these subjects' miss responses received false positive feedback (S, strict condition). Thus, the order of manipulation was feedback encouraging lax, neutral, and strict (LNS group) responding. This order was reversed for the other half of the subjects (strict, neutral, and lax, SNL group).

\section{Results and Discussion}

$\operatorname{Accuracy}\left(\mathbf{A}_{\mathbf{z}}\right)$

An ANOVA for $\mathrm{A}_{\mathrm{z}}$ with factors of group (LNS or SNL) and test (first, second, or third) yielded a significant main effect only of test $\left[F(2,54)=5.01, M S_{\mathrm{e}}=0.01, p<.05\right]$. In general, $A_{z}$ decreased across tests. Post hoc Tukey's HSD comparisons demonstrated that accuracy was similar and higher in the first and second test in comparison with the final tests $(p<.005)$. Importantly, there was no evidence for an interaction between group and test $(p>$ .30). Thus, although performance generally declined, the groups displayed similar accuracy within each separate test (see Table 1). Given this fact, contrasts of criterion across the groups for each separate test are not confounded with notable accuracy differences.

\section{Decision Criteria $\left(c_{a}\right)$}

To analyze the effects of the feedback manipulation, we focused on the old/new decision criterion (Table 1) using a two-way ANOVA with the factors of group (LNS or SNL) and test (first, second, or third). For the omnibus and post hoc analyses, 1 subject was excluded due to an extreme estimate of old item standard deviation $(>4$ for one of the three tests) that likely rendered the criterion estimates for this subject unreliable.

The analysis revealed a main effect of group $[F(1,27)=$ $\left.6.25, M S_{\mathrm{e}}=1.18, p<.05\right]$, with the SNL group demonstrating a generally more conservative criterion (mean $\mathrm{c}_{\mathrm{a}}=.24$ ) than that of the LNS group (mean $\mathrm{c}_{\mathrm{a}}=.01$ ). There was also a main effect of test, which suggests that the criterion generally became more lax across the three sequential tests $\left[F(2,54)=6.06, M S_{\mathrm{e}}=0.18, p<.005\right]$. Finally, there was a significant group $\times$ test interaction $\left[F(2,54)=10.83, M S_{\mathrm{e}}=0.31, p<.001\right]$, which indicates that the group differences in criterion were nonuniform across the tests (Table 1). Simple $t$ tests across the groups for each test demonstrated that the criterion was significantly more conservative in the SNL group during Test $1[t(27)=3.07, p<.005]$, and it remained so during

Table 1

Experiment 2 Unequal Variance Accuracy and Decision Criterion Estimates Across Groups and Tests

\begin{tabular}{|c|c|c|c|c|c|c|c|c|c|c|c|c|}
\hline & \multicolumn{6}{|c|}{ Lax-Neutral-Strict } & \multicolumn{6}{|c|}{ Strict-Neutral-Lax } \\
\hline & \multicolumn{2}{|c|}{ Test 1} & \multicolumn{2}{|c|}{ Test 2} & \multicolumn{2}{|c|}{ Test 3} & \multicolumn{2}{|c|}{ Test 1} & \multicolumn{2}{|c|}{ Test 2} & \multicolumn{2}{|c|}{ Test 3} \\
\hline & $M$ & $S D$ & $M$ & $S D$ & $M$ & $S D$ & $M$ & $S D$ & $M$ & $S D$ & $M$ & $S D$ \\
\hline Hit & .78 & .11 & .81 & .09 & .74 & .12 & .60 & .19 & .65 & .13 & .71 & .11 \\
\hline False alarm & .21 & .13 & .24 & .12 & .23 & .13 & .14 & .08 & .19 & .08 & .29 & .07 \\
\hline $\mathrm{A}_{\mathrm{z}}$ & .85 & .08 & .86 & .06 & .82 & .07 & .78 & .12 & .81 & .07 & .77 & .08 \\
\hline$\sigma_{\text {old }}$ & 1.34 & 0.67 & 1.35 & 0.67 & 1.42 & 0.86 & 1.72 & 0.80 & 1.33 & 0.37 & 1.12 & 0.21 \\
\hline$\underline{\mathrm{c}_{\mathrm{a}} \text { overall }}$ & .02 & .31 & -.06 & .24 & .06 & .29 & .39 & .35 & .27 & .29 & .05 & .24 \\
\hline
\end{tabular}

Note $-\sigma_{\text {old }}$, estimated standard deviations of old item distribution; $c_{a}$ overall, criteria for the overall "old/new" response. 
Test 2 , in which all feedback was fully correct (i.e., neutral condition) $[t(27)=3.36, p<.005]$. However, the overall old/new criterion did not differ for the groups in the final test, in which the biasing manipulation was reversed $[t(27)<1$, n.s. $]$.

Overall, the analysis of the accuracy and old/new criterion statistics suggested that the manipulation differentially altered the location of the overall old/new decision criterion across the two groups without producing notable differences in accuracy across the groups. Importantly, the induced relative difference in criterion that occurred across the groups during the first test was carried over into the second test, despite the removal of the biasing manipulation and the presence of entirely correct feedback. It is important to emphasize that this means that the response tendency or preference acquired during Test 1 survived an intermediate study period and was expressed in Test 2 , even though it was no longer fully supported by the feedback procedure. This suggests a durable form of learning that is inconsistent with an explicit strategy that would have presumably been abandoned in Test 2 , as soon as the feedback indicated that it was no longer working or necessary.

The current procedure did not produce a significant criterion difference across the groups in Test 3, which suggests that the reversal of the feedback conditions effectively eliminated the relative differences in response preferences that were acquired in Test 1 and sustained during Test 2. The data in Table 2, however, suggest that the changes in criterion across tests within each group were dissimilar. More specifically, although the SNL group demonstrated a large shift in the criterion between Test 2 and Test 3 (.27 to .05), the LNS group displayed a much more modest change ( -.06 to .06$)$; this change in the LNS group was statistically reliable $[t(14)=2.63, p<.05]$. However, interpreting these differences in the relative size of the criterion changes is complicated because as noted above, the accuracy of the groups also generally declined in the transition between Tests 2 and 3. Under such situations, criterion estimates can be artificially masked or enhanced (Pastore et al., 2003), and thus comparisons of criterion (or criterion change) across conditions in which accuracy also changes should generally be avoided. In Experiment 3 , we sizably reduced the number of trials per test in hopes of preventing a decline in accuracy. Because clearly disproportionate differences in the criterion change rates were not observed in Experiment 3 (see below), and accuracy was also unchanged across tests, we do not consider further the differences noted above.

As with Experiment 1, the design also allowed for ROC analysis. In order to avoid complications arising from the sequential testing and the general decline of performance noted above, we focused entirely on the first test across the two subject groups when considering the ROCs. The general prediction, given the unequal variance signal detection model shown in Figure 1, is that both groups should lie along the same ROC surface and differ only in the location of one or more of their cumulated response points. Unexpectedly, visual inspection of the aggregate ROC suggested instead that the ROC of the strict group was more asymmetrical and appeared slightly lower than that of the lax group, beginning at the fourth ROC point (Figure 3A).

If the visual impression of the aggregate ROC is reliable in the individual subject data, then the estimate of old item standard deviation provided by the fitting procedures should also differ systematically for the groups. The estimates of the old item standard deviation marginally differed across the groups, suggesting that the ROC of the strict group was slightly more asymmetric than that of the lax group $[t(25)=1.41, p=.054]$ during Test 1 .

In their entirety, the results of Experiment 2 yielded four important results: (1) The false feedback manipulation clearly shifted the relative position of the old/new criterion across the groups; (2) the relative shift in the group's criterion was sustained for a considerable period, even when the manipulation was removed and feedback was unbiased (Test 2); (3) reversing the feedback manipulation eliminated or lessened the difference across the groups (Test 3); and (4) during Test 1, when the criterion/decision group differences were most pronounced, there was also a slight difference in the shape of the observed ROC across the groups, with conservative responding linked to more asymmetric ROCs. This latter finding potentially converges with earlier work by Van Zandt (2000) that demonstrates that inducing an increasingly conservative basis for responding via instruction or payoff contingencies can yield increasingly asymmetric ROCs. We save further consideration of this potential asymmetry difference until the General Discussion. Finally, it should be noted that the criterion shifts are occurring despite the fact that the manipulation only occurs, at least initially, on a minority of trials. That is, because it is restricted to misses and false alarms, it is a fairly subtle manipulation; nonetheless, the group differences were prominent.

Table 2

Experiment 3 Accuracy and Decision Criterion Estimates Across Groups and Tests

\begin{tabular}{|c|c|c|c|c|c|c|c|c|c|c|c|c|}
\hline & \multicolumn{6}{|c|}{ Lax-Strict-Strict } & \multicolumn{6}{|c|}{ Strict-Lax-Lax } \\
\hline & \multicolumn{2}{|c|}{ Test 1} & \multicolumn{2}{|c|}{ Test 2} & \multicolumn{2}{|c|}{ Test 3} & \multicolumn{2}{|c|}{ Test 1} & \multicolumn{2}{|c|}{ Test 2} & \multicolumn{2}{|c|}{ Test 3} \\
\hline & $M$ & $S D$ & $M$ & $S D$ & $M$ & $S D$ & $M$ & $S D$ & $M$ & $S D$ & $M$ & $S D$ \\
\hline Hit & .78 & .08 & .70 & .13 & .68 & .16 & .72 & .15 & .77 & .11 & .81 & .12 \\
\hline False alarm & .20 & .10 & .13 & .08 & .14 & .08 & .15 & .09 & .22 & .13 & .26 & .15 \\
\hline $\mathrm{A}_{\mathrm{z}}$ & .86 & .06 & .85 & .05 & .81 & .11 & .86 & .09 & .85 & .10 & .86 & .09 \\
\hline$\sigma_{\text {old }}$ & 1.22 & 0.33 & 1.23 & 0.58 & 1.56 & 1.08 & 1.42 & 0.33 & 1.15 & 0.60 & 1.05 & 0.41 \\
\hline$c_{a}$ overall & .05 & .24 & .29 & .31 & .26 & .29 & .19 & .26 & .00 & .24 & -.09 & .28 \\
\hline
\end{tabular}

Note $-\sigma_{\text {old }}$, estimated standard deviations of old item distribution; c $c_{a}$ overall, criteria for the overall "old/new" response. 
A

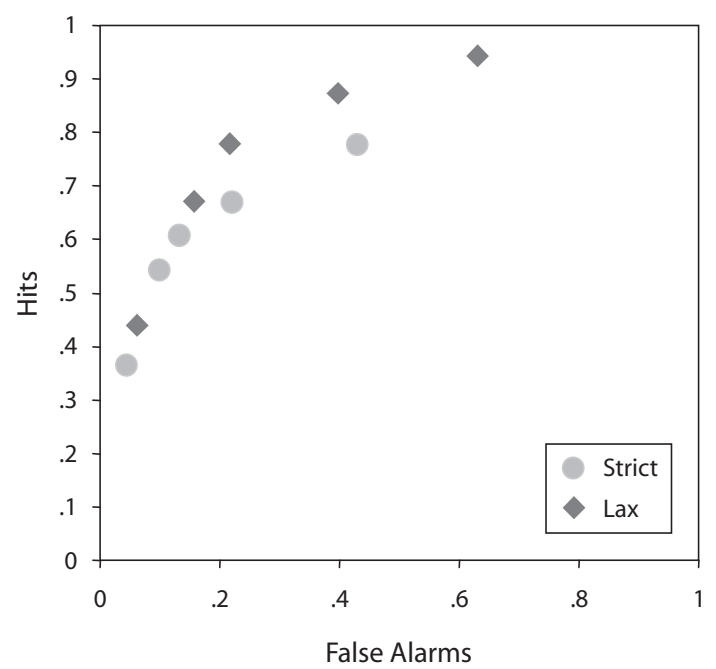

B

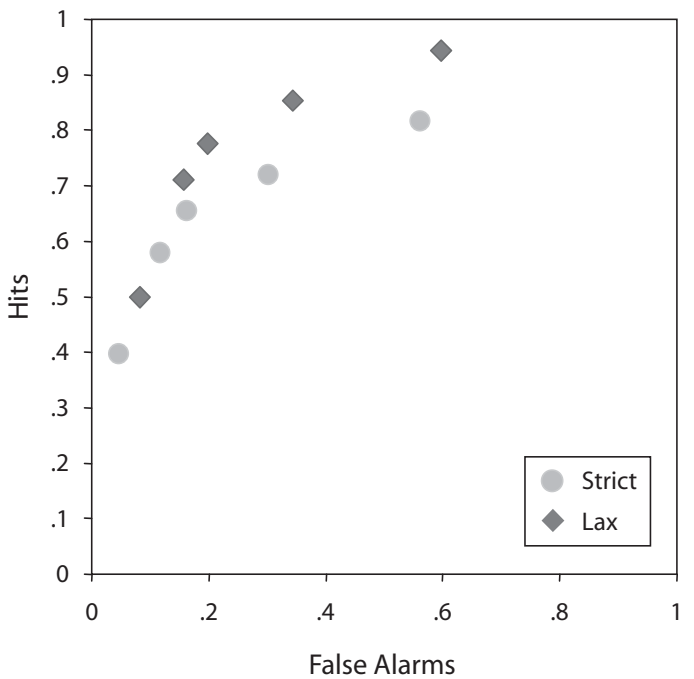

Figure 3. Observed aggregate recognition memory ROCs from Experiment 2 (A) and Experiment 3 (B) during Test 1 of each experiment.

\section{EXPERIMENT 3 Biased-Feedback Procedure (Old/New Recognition: One-Step Classification)}

The results of Experiment 2 demonstrate that there is considerable lability in the location of the old/new decision criterion, as a function of the biased-feedback manipulation. During the test, subjects first classified each item as old or new, and only after doing so did they report confidence. Although this two-step procedure is common, some researchers have suggested that it induces a secondary response/rating demand that is not present when confidence and classification are instead indicated via a single response on each trial (Galvin, Podd, Drga, \& Whitmore, 2003). Under a one-step procedure, subjects jointly indicate not only whether they believe the item to be old or new, but also their confidence. In Experiment 3, we used this one-step response procedure to verify that the old/new criterion lability observed in Experiment 2 would remain under this different response format.

The second goal of Experiment 3 was to see whether the criterion shifts demonstrated during Test 1 in Experiment 2 could be completely reversed across the subject groups. More specifically, in Experiment 2, by the final test, there was no apparent difference between the overall $c_{a}$ estimates of the two groups. We reasoned that this occurred because the reversed-feedback contingencies in Test 3 had to overcome the previously shifted criterion locations established in Test 1 and maintained throughout Test 2; however, a stronger case for a labile decision criterion could be made if a crossover of $c_{a}$ values was observed for the groups. To encourage this, we replaced the neutral-feedback condition of the second test with one encouraging the same bias as the final test, since this procedure allows more opportunity to learn the reversed contingencies of biased feedback during last two test runs. Thus, the group starting with feedback-encouraging lax responding in Test 1 received two additional subsequent tests encouraging strict responding (lax, strict, strict, LSS). The reverse order was administered to the second group (strict, lax, lax, SLL).

Additionally, in Experiment 3 we introduced a postexperiment questionnaire to determine whether subjects were generally aware that the feedback was not entirely accurate, and if so, to determine whether they were aware of the manner in which the feedback was inaccurate. Finally, we also considerably reduced the number of test trials in Experiment 3. This was done in an attempt to lessen fatigue and perhaps prevent the general decline in performance observed in Experiment 2.

\section{Method}

\section{Subjects}

Thirty-four Duke University undergraduates participated in return for partial course credit, and two separate groups of 17 were included in the analyses. Informed consent was obtained, as required by the human subjects review committee of Duke University. Subjects were randomly assigned to separate feedback conditions that differed in the order of the feedback manipulation across tests. Five subjects were removed and replaced for a combination of poor performance $\left(d^{\prime}<0.5\right)$ and an indication that they were potentially aware of the manipulation on a postexperiment questionnaire. ${ }^{2}$

\section{Procedure}

A total of 360 nouns were drawn randomly for each subject from the same item pool we used in Experiments 1 and 2. From this total, three lists of 120 items (60 old, 60 new items, for each cycle) were constructed for use in three sequential study/test cycles. During testing, the items were presented in the center of the screen, located above a one-step response cue: "Is this OLD or NEW? NEW: SureProbably-Guess | Guess-Probably-Sure: OLD.” There were six prespecified keys on the keyboard with red identifying stickers. For 
half the subjects, the location of the OLD and NEW buttons was counterbalanced with the presentation of a cue: "Is this NEW or OLD? OLD: Sure-Probably-Guess | Guess-Probably-Sure: NEW." Responding was self-paced. Other than these changes, the procedure was identical to that in Experiment 2.

\section{Results and Discussion}

\section{Accuracy $\left(\mathbf{A}_{\mathrm{z}}\right)$}

A two-way ANOVA for $A_{z}$ with the factors of group (LSS or SLL) and test (first, second, or third) yielded no significant effects (Table 2).

\section{Decision Criteria $\left(\mathbf{c}_{\mathrm{a}}\right)$}

For the omnibus ANOVA and post hoc analyses, one subject who had extreme old item standard deviations estimates ( $>4$ for two of the three tests) was removed, which likely rendered his or her $\mathrm{c}_{\mathrm{a}}$ estimates unreliable.

For the old/new criterion, the ANOVA yielded a main effect of group $\left[F(1,31)=4.45, M S_{\mathrm{e}}=0.67, p<.05\right]$ with the LSS group (mean $\left.c_{a}=.20\right)$ being generally more conservative than the SLL group (mean $\mathrm{c}_{\mathrm{a}}=.04$ ). There was no main effect of test $(p<.45)$, but the interaction between group and test was again significant $[F(2,62)=$ $\left.15.85, M S_{\mathrm{e}}=0.59, p<.001\right]$, which indicates that the relative group differences in criterion changed across the tests. Post hoc $t$ tests demonstrated that although the difference was in the right direction, the criteria did not significantly differ during Test $1(p<.12)$. The SLL group, however, was significantly more liberal than the LSS group during Test $2[t(31)=2.87, p<.05]$, in which the feedback contingencies were reversed and remained so during Test $3[t(31)=3.51, p<.01]$. These relative criterion differences were observed in a pool of subjects whose posttest questionnaires indicated they were unaware of any abnormalities or systematic inaccuracies in the feedback. We classified subjects as aware if they indicated in any manner that they believed the feedback to be inaccurate or skewed in response to the question "Did you find the feedback to your 'OLD' or 'NEW' answer helpful? If not, please specifically explain why."

Not only did the subjects who were included in the analysis indicate that they were unaware of the manipulation, but also those who instead reported that there was something odd or otherwise inconsistent about the feedback appeared to respond in a qualitatively different manner than those who reported being unaware. More specifically, we removed five subjects from Experiment 3: One misunderstood the task and four reported feeling that the feedback was inaccurate for a particular response type. By the last test, these aware subjects showed abnormally decreased accuracy (mean $d^{\prime}=0.22$ ) and abnormally rapid reaction times $($ mean $=555 \mathrm{msec})$. This suggests that these subjects noticed the manipulation and chose to respond by simply pressing the key that rarely led to an "incorrect" feedback outcome. Since they were not actually processing the memoranda, their accuracy fell to near chance, and their reaction times were unreasonably rapid.

Although the analysis of the area under ROCs $\left(\mathrm{A}_{\mathrm{z}}\right)$ suggested that there were no accuracy differences across the groups, visual inspection of the aggregate ROC for
Test 1 again suggested that the ROC for the strict group was slightly more asymmetrical than that of the lax group (Figure 3B). Using the estimates obtained from the fitting, we statistically examined this visual impression at the level of individual subjects. As in Experiment 2, the difference in the estimates of the old item variability approached significance $[t(31)=1.80, p=.081]$, with results that suggest a greater asymmetry between old and new variance for the strict versus the lax group.

In summary, the findings of Experiment 3 reinforced those of Experiment 2. For the overall old/new criterion, the $\mathrm{c}_{\mathrm{a}}$ estimate of criterion was clearly and systematically affected by the biased-feedback manipulation, despite the different response procedure. These adaptive decision criterion shifts occurred without a concomitant change in accuracy across groups, as estimated by $\mathrm{A}_{\mathrm{z}}$, and were accompanied by slight evidence that strict responding is associated with a more asymmetrical ROC. Although numerically consistent with a full crossover of $c_{a}$ scores, the groups did not significantly differ in criterion on the first test. This might reflect the differences in test list length used in the two studies, with Experiment 2 having a list length of 200 and Experiment 3 a length of 120 . Given that the effect is a learning phenomenon, group differences may be more robust following longer test lists, which increase exposure to the biasing feedback. Nonetheless, the data demonstrate that both response formats (two-step and one-step) are influenced by the false-feedback procedure, and the postexperiment questionnaire data further suggest that subjects are, at least at the basic self-report level, unaware of the nature of the experimental manipulation.

\section{GENERAL DISCUSSION}

The present study explored the lability of recognition decision criteria during the course of testing. As noted in the introduction, although pretest instructions effectively induce criterion shifts during recognition memory, there is considerable evidence that criterion placement is rigid once testing sessions begin. In studies that demonstrate this rigidity, large and prominent changes in the density of targets, the level of memory evidence associated with prominent categories of items, and the average strength of targets have failed to induce criterion shifts. This suggests that a key distinction exists between subjects who have been provided explicit instructions that guide response strategies and subjects with the ability to inductively use memory-linked list regularities during testing in order to adopt adaptive criterion strategies. Furthermore, on the rare occasions in which criterion shifts were induced during testing, these shifts required large, systematic manipulations of the memory stimuli, coupled with feedback that likely led subjects to become explicitly aware of the stimulus manipulations.

For example, in Verde and Rotello (2007), there was a large change in the target strength across test halves, and subjects received both trial-by-trial feedback and performance summaries for every quartile of the test during which testing was interrupted and tallies of their correct and incorrect responses for that quartile were presented. 
These performance summaries are arguably analogous to providing subjects explicit instructions to become more or less cautious, and they likely make the target strength manipulation explicit to a majority of the subjects. Similarly, Rhodes and Jacoby (2007) also demonstrated feedbackinduced criterion shifts during testing by linking quadrants of the test screen to different target presentation probabilities. However, the shifts were most evident for subjects who were explicitly aware of the relationship between quadrant and probability, and the effect was markedly diminished when feedback was removed. Coupled with the findings of Estes and Maddox (1995), in which the large base rate differences were not effective until subjects also received feedback for number and digit strings (although not for words), the previous work suggests that veridical feedback may induce criterion shifts only when coupled with systematic manipulations of the test probes. Under such circumstances, the major role of the feedback may largely be to make it increasingly likely that subjects will become explicitly aware of the stimulus manipulation.

Critically then, there exist no demonstrated cases of criterion shifts in the absence of prominent manipulations of the test materials and likely explicit awareness of those manipulations. This is important because the typical characterizations of signal detection theory assume a highly flexible and adaptive criterion that should be adjustable, even without prominent changes in the underlying evidence. Such a criterion would allow the subject to take advantage of changing environmental contingencies in order to capitalize, for example, on changing reward contingencies (Curran, DeBuse, \& Leynes, 2007; Macmillan \& Creelman, 1991). Using a biased-feedback technique that provides subjects with trial-specific information about the appropriateness of the currently held criterion placement, Experiments 2 and 3 fill this gap by demonstrating an adaptive criterion that subjects flexibly adjust independently of the nature of the available underlying memory evidence upon which the criterion operates.

Although standard signal detection theory assumes that the decision operation is functionally independent of observer accuracy - something that our findings also support — one might assume that shifts in criterion would be more likely when sensitivity is low. For instance, the null findings in the word recognition condition of Estes and Maddox (1995) might have been due to subjects' higher accuracy during word recognition in comparison with their performance in the nonverbal recognition conditions, in which accuracy was considerably lower and criterion shifts were readily observed. We tested this theory by first correlating the tendency to shift criterion and accuracy in Experiments 2 and 3. For this, the criterion difference between Test 1 and Test 3 was correlated with observer sensitivity in the initial test block. This correlation was not significant in either experiment $(r=-.22$ and $r=.01$ ), which indicates that sensitivity to the feedback manipulation was not closely linked with discrimination skill. Additionally, a median split of high versus low performers yielded a similar conclusion, with high- and low-performing groups replicating the group $\times$ test interactions of criterion in Experiment $2[F(2,22)=5.36, p<$
.05 , and $F(2,28)=4.48, p<.05$, for low and high performers, respectively $]$ and Experiment $3[F(2,28)=7.62$, $p<.01$, and $F(2,32)=10.23, p<.001$, for low and high performers, respectively]. Thus, the pattern of criterion changes in response to the biased feedback occurs both for high- and low-performing groups.

\section{Is Criterion Learning Implicit?}

Aside from demonstrating a flexible or adaptive criterion that operates independently of stimulus evidence during testing, the present study provides initial evidence that the induced criterion change may reflect a type of implicit learning that is perhaps analogous to that which occurs during probabilistic classification learning paradigms (Gluck \& Bower, 1988; Knowlton et al., 1994). In support of this implicit learning interpretation, the feedback questionnaire data in Experiment 3 indicated that subjects included in the analyses reported being unaware of anything odd or skewed about the feedback, and those excluded for reporting anomalies in the feedback also displayed qualitatively different response patterns from the unaware group. The latter appeared to adopt a response strategy similar to the "maximizing" strategies sometimes observed in studies of the matching law - strategies that serve to maximize the number of positive outcomes (Herrnstein, 1970). However, it must also be noted that these subjects may have decided to respond in this manner simply to end the testing session as quickly as possible, and not necessarily to maximize positive feedback outcomes.

A second line of evidence that suggests an implicit mechanism was the demonstration that the shifted criterion was preserved when the biased feedback reverted to fully correct (Test 2, Experiment 2). More specifically, in Experiment 2, subjects received fully correct feedback after the first test that successfully induced a betweengroup criterion difference, and this criterion difference remained during the second test, despite the fact that all of the feedback was now correct. Thus, the subjects underwent an intervening study period and a subsequent test with fully correct feedback, yet they still displayed different response criteria across the groups. If the subjects were using an explicit strategy and monitoring the feedback for the utility of this strategy, it would seem more likely that it would have been quickly abandoned during the second test, when the fully correct feedback should have alerted them to its inapplicability. Partially supporting this interpretation, Rhodes and Jacoby (2007) demonstrated that removal of feedback, for subjects aware of their manipulation of target probabilities, eliminated the criterion shifts induced by this manipulation. Thus, the shift in criterion depended upon continued support of an explicit strategy provided via the feedback. In the present study, we suggest that the different positive and negative feedback tendencies induced by the biased-feedback procedure altered the preferences for the two response types (old and new). Because apparently similar forms of response learning are prevalent across species incapable of forming explicit strategies, it seems reasonable to speculate that some form of response reinforcement learning plays a role in the induced criterion shifts observed here. 
This would be highly exciting, since it might reflect an interaction between a more implicit procedural system and an explicit learning/memory system (Poldrack et al., 2001). Indeed, although they were unsuccessful in inducing a criterion shift for verbal recognition stimuli, Estes and Maddox (1995) invoked a model with two separate learning processes for their successfully induced criterion shifts in digit and string recognition. Whereas one process elicited a similarity-recognition value for memoranda, the other process was a learning mechanism that tracked the feedback ratios associated with the difference response types old and new, and this information was incorporated whenever stimulus recognition was uncertain.

Although we feel that the questionnaire data and the pattern of responding are consistent with a response reinforcement learning phenomenon that we have tentatively termed implicit, it is important to note that the primary purpose of the present investigation was to demonstrate a flexible and adaptive criterion during testing that did not rely upon gross manipulations of the memoranda or upon explicit biasing instructions. Nonetheless, the present data suggest that further investigation of the characteristics and neural substrates of biased-feedback criterion learning are warranted because the shifts demonstrated here may be fundamentally different from those obtained through explicitly warning subjects to be cautious or lax in responding.

\section{A Change in the Quality of Information Used?}

One unexpected but interesting finding in the present series of studies was the small, but somewhat consistent, evidence that changes in the criterion influenced the shape of the observed ROC. Across Experiments 2 and 3 the evidence suggests that subjects who were more conservative evidenced a greater asymmetry in the old/new recognition ROC. At present, we can only speculate on the potential cause of this asymmetry, given that only one other study appears to have reliably demonstrated a similar phenomenon, and that the effect was small and difficult to detect here. In two recent experiments, Van Zandt (2000) used payoff matrices and changes in target frequencies (explicitly warned prior to each test) to manipulate the response criterion. Overall, the data demonstrated that increasingly conservative responding was associated with more asymmetrical ROCs; this was modeled with a two-counter race model. The key aspect of the model that accommodated the shape differences was that it assumed that reaching the threshold to decide that an item was either old or new relied on different response counters for evidence of oldness or newness. These counters, each having a separate decision criterion, keep track of memory evidence that favors old or new responses, and the difference in evidence of these counters transforms into a confidence rating. Using this model, favoring one counter or the other leads to asymmetric ROCs. However, direct comparison of the Van Zandt study and the present study is risky for several methodological reasons. For example, in Van Zandt's study, test lists were much shorter, and encoding duration was also limited $(750 \mathrm{msec})$. Furthermore, subjects had extensive training, participating in 12 study/test blocks per daily session, and 10 sessions spread out over 2 weeks.
Nonetheless, linking changes in shape of the ROC to differences in the manner in which subjects parse the accrual of separate forms of evidence may be a promising method of explaining the present ROC data.

Another possible explanation of the change in the shape of the ROC may rely upon multidimensional memory representations with different retrieval orientations modeled as different decision axes. Under this approach, subjects may change the mixture of types of information when they become more conservative (see Banks, 2000; see also Johnson, Hashtroudi, \& Lindsay, 1993). For example, if one further assumed that inducing conservative responding caused subjects to rely upon more detailed contextual source memory, then a multidimensional model could potentially accommodate a change in the asymmetry of the ROC by shifting the decision axis within a stationary evidence space. A fuller investigation of this and other possibilities is beyond the scope of the present study, although it may be a promising future avenue.

\section{Summary}

In summary, the present study demonstrates that independent of the actual strength or memory evidence available for the test items, subjects are easily and durably influenced by subtle differences in the validity of error-related feedback during recognition, which leads to prominent and sustained shifts in the measured decision criterion. The subjects report being unaware of the biased nature of the manipulation that induces the shift, and the response tendency is maintained for some time, even when the manipulation is removed. This is the first time such a feedback procedure has been shown to be effective in criterion learning during explicit recognition. Whether this learning-based shift is related to more explicit instructionbased manipulations of criterion or whether it instead reflects a more implicit form of classification or response reinforcement learning remains an important topic for future research. Several aspects of the present data, however, suggest the latter to be true. If correct, then this in turn suggests that criterion shifts achieved through explicit instruction and those achieved through this type of biasedfeedback learning may rely on at least partially different neural substrates. Regardless, the data converge with prior reports that demonstrate that subjects are quite sensitive to trial-specific information that is relevant for assessing the suitability of currently held memory decision criteria (Benjamin, 2003; Dobbins \& Kroll, 2005).

\section{AUTHOR NOTE}

This research was supported by NIH Grant R01-MH073982-01. We thank Julie Grimley for help with data collection. Correspondence regarding this article should be sent to S. Han, Department of Psychology \& Neuroscience, Duke University, Durham, NC 27708-0086 (e-mail: sanghoon.han@duke.edu).

\section{REFERENCES}

Azimian-Faridani, N., \& Wilding, E. L. (2006). The influence of criterion shifts on electrophysiological correlates of recognition memory. Journal of Cognitive Neuroscience, 18, 1075-1086.

BAnKs, W. P. (2000). Recognition and source memory as multivariate decision processes. Psychological Science, 11, 267-273. 
Benjamin, A. S. (2003). Predicting and postdicting the effects of word frequency on memory. Memory \& Cognition, 31, 297-305.

BeNJAMIN, A. S., \& BAWA, S. (2004). Distractor plausibility and criterion placement in recognition. Journal of Memory \& Language, 51, 159-172.

BoHIL, C. J., \& MADDox, W. T. (2003). On the generality of optimal versus objective classifier feedback effects on decision criterion learning in perceptual categorization. Memory \& Cognition, 31, 181-198.

Brown, J., LewIs, V., \& Monk, A. F. (1977). Memorability, word frequency, and negative recognition. Quarterly Journal of Experimental Psychology, 29, 461-473.

Carterette, E. C., Friedman, M. P., \& Wyman, M. J. (1966). Feedback and psychophysical variables in signal detection. Journal of the Acoustical Society of America, 39, 1051-1055.

Curran, T., DeBuse, C., \& Leynes, P. A. (2007). Conflict and criterion setting in recognition memory. Journal of Experimental Psychology: Learning, Memory, \& Cognition, 33, 2-17.

Dobbins, I. G., \& Kroll, N. E. A. (2005). Distinctiveness and the recognition mirror effect: Evidence for an item-based criterion placement heuristic. Journal of Experimental Psychology: Learning, Memory, \& Cognition, 31, 1186-1198.

Dorfman, D. D., \& Biderman, M. (1971). A learning model for a continuum of sensory states. Journal of Mathematical Psychology, 8 264-284.

Elliott, R., Frith, C. D., \& Dolan, R. J. (1997). Differential neural response to positive and negative feedback in planning and guessing tasks. Neuropsychologia, 35, 1395-1404.

Estes, W. K., \& MADDOX, W. T. (1995). Interactions of stimulus attributes, base rates, and feedback in recognition. Journal of Experimental Psychology: Learning, Memory, \& Cognition, 21, 1075-1095.

Friedman, M. P., Carterette, E. C., \& Nakatani, L. (1968). Comparisons of some learning models for response bias in signal detection. Perception \& Psychophysics, 3, 5-11.

Galvin, S. J., Podd, J. V., Drga, V., \& Whitmore, J. (2003). Type 2 tasks in the theory of signal detectability: Discrimination between correct and incorrect decisions. Psychonomic Bulletin \& Review, 10, 843-876.

Glanzer, M., \& Adams, J. K. (1990). The mirror effect in recognition memory: Data and theory. Journal of Experimental Psychology: Learning, Memory, \& Cognition, 16, 5-16.

GLUCK, M. A., \& Bower, G. H. (1988). From conditioning to category learning: An adaptive network model. Journal of Experimental Psychology: General, 117, 227-247.

Herrnstein, R. J. (1970). On the law of effect. Journal of the Experimental Analysis of Behavior, 13, 243-266.

Hirshman, E., \& HenZler, A. (1998). The role of decision processes in conscious recollection. Psychological Science, 9, 61-65.

Johnson, M. K., Hashtroudi, S., \& Lindsay, D. S. (1993). Source monitoring. Psychological Bulletin, 114, 3-28.

Knowlton, B. J., Squire, L. R., \& GlucK, M. A. (1994). Probabilistic classification learning in amnesia. Learning \& Memory, 1, 106-120.

Kubovy, M., \& Healy, A. F. (1977). The decision rule in probabilistic categorization: What it is and how it is learned. Journal of Experimental Psychology: General, 106, 427-446.

Macmillan, N. A., \& Creelman, C. D. (1991). Detection theory: A user's guide. New York: Cambridge University Press.

Maddox, W. T., \& Bohil, C. J. (2001). Feedback effects on costbenefit learning in perceptual categorization. Memory \& Cognition, 29, 598-615

Morrell, H. E. R., Gaitan, S., \& Wixted, J. T. (2002). On the nature of the decision axis in signal-detection-based models of recognition memory. Journal of Experimental Psychology: Learning, Memory, \& Cognition, 28, 1095-1110.

Papo, D., Baudonnière, P.-M., Hugueville, L., \& Caverni, J.-P. (2003). Feedback in hypothesis testing: An ERP study. Journal of Cognitive Neuroscience, 15, 508-522.

Pashler, H., Cepeda, N. J., Wixted, J. T., \& Rohrer, D. (2005). When does feedback facilitate learning of words? Journal of Experimental Psychology: Learning, Memory, \& Cognition, 31, 3-8.

Pastore, R. E., Crawley, E. J., Berens, M. S., \& Skelly, M. A. (2003). "Nonparametric" $A$ ' and other modern misconceptions about signal detection theory. Psychonomic Bulletin \& Review, 10, 556-569.

Poldrack, R. A., Clark, J., Paré-Blagoev, E. J., Shohamy, D., Moy-
ANo, J. C., Myers, C., \& Gluck, M. A. (2001). Interactive memory systems in the human brain. Nature, 414, 546-550.

RatclifF, R., Sheu, C.-F., \& Gronlund, S. D. (1992). Testing global memory models using ROC curves. Psychological Review, 99, 518-535.

Rhodes, M. G., \& JACOBY, L. L. (2007). On the dynamic nature of response criterion in recognition memory: Effects of base rate, awareness, and feedback. Journal of Experimental Psychology: Learning, Memory, \& Cognition, 33, 305-320.

Rotello, C. M., Macmillan, N. A., Reeder, J. A., \& Wong, M. (2005). The remember response: Subject to bias, graded, and not a process-pure indicator of recollection. Psychonomic Bulletin \& Review, 12, 865-873.

SHEPARD, R. N. (1967). Recognition memory for words, sentences, and pictures. Journal of Verbal Learning \& Verbal Behavior, 6, 156-163.

Strack, F., \& Förster, J. (1995). Reporting recollective experiences: Direct access to memory systems? Psychological Science, $\mathbf{6}$, 352-358.

Stretch, V., \& Wixted, J. T. (1998). On the difference between strength-based and frequency-based mirror effects in recognition memory. Journal of Experimental Psychology: Learning, Memory, \& Cognition, 24, 1379-1396.

Thomas, E. A. C. (1973). On a class of additive learning models: Errorcorrecting and probability matching. Journal of Mathematical Psychology, 10, 241-264.

Treisman, M., \& Williams, T. C. (1984). A theory of criterion setting with an application to sequential dependencies. Psychological Review, 91, 68-111.

VAN ZANDT, T. (2000). ROC curves and confidence judgments in recognition memory. Journal of Experimental Psychology: Learning, Memory, \& Cognition, 26, 582-600.

Verde, M. F., \& Rotello, C. M. (2007). Memory strength and the decision process in recognition memory. Memory \& Cognition, 35, 254-262.

Wallace, W. P. (1982). Distractor-free recognition tests of memory. American Journal of Psychology, 95, 421-440.

Wallace, W. P., SAWYer, T. J., \& RoberTson, L. C. (1978). Distractors in recall, distractor-free recognition, and the word-frequency effect. American Journal of Psychology, 91, 295-304.

White, K. G., \& WiXted, J. T. (1999). Psychophysics of remembering. Journal of the Experimental Analysis of Behavior, 71, 91-113.

Wixted, J. T., \& STRETch, V. (2000). The case against a criterion-shift account of false memory. Psychological Review, 107, 368-376.

Yonelinas, A. P., Dobbins, I. [G.], Szymanski, M. D., Dhaliwal, H. S., \& KING, L. (1996). Signal-detection, threshold, and dualprocess models of recognition memory: ROCs and conscious recollection. Consciousness \& Cognition, 5, 418-441.

\section{NOTES}

1. Visual and statistical inspections of the shape of ROCs indicate that the recognition data in the present experiment do not follow the equal variance signal detection model assumption. We employed $\mathrm{A}_{\mathrm{z}}$ and $\mathrm{c}_{\mathrm{a}}$, since the assumption of the unequal variance signal detection (UEVSD) model renders invalid the use of the equal variance signal detection measures of accuracy/bias - such as $d^{\prime}$ or c - although they resulted in a similar pattern of results. $A_{z}$, the area under ROCs assuming normal evidence distributions, provides a single value of accuracy, even when the old and new item distributions do not share the same variance. $c_{a}$ is a bias statistic that indicates the criterion location in the units of rootmean-square standard deviation of the old and new item distributions, and, thus, it can also be used in cases where the distribution variances are thought to be unequal (see Macmillan \& Creelman, 1991). To provide an estimate of the relative variance difference for the old and new item distributions, we individually fit the UEVSD model to each subject's data using Excel's Solver routine by minimizing the sum of squared errors of prediction for old and new response proportions at each criterion level. During fitting, each subject's new item distribution standard deviation was fixed to 1, and the Solver algorithm proceeded to adjust the distance between the distributions $(d)$, the five decision criteria, and the standard deviation of the old item distribution in order to minimize the sum of squared errors of prediction. Importantly, unlike a linear regression, this method assumes error in both old and new item response propor- 
tions (for details, see Yonelinas, Dobbins, Szymanski, Dhaliwal, \& King, 1996). Following the fit, the obtained variance estimates were used to calculate $\mathrm{A}_{\mathrm{z}}$ and $\mathrm{c}_{\mathrm{a}}$ using the following formulas:

$$
\begin{gathered}
\mathrm{A}_{\mathrm{z}}=\Phi\left(\frac{\mu_{\text {old }}}{\sqrt{1+\sigma_{\text {old }}^{2}}}\right) \\
\mathrm{c}_{\mathrm{a}}=\frac{-\sqrt{2} s}{\left(1+s^{2}\right)^{\frac{1}{2}}(1+s)}[z(H)+z(F)],
\end{gathered}
$$

where $\Phi$ indicates the transformation of the value by the unit-normal distribution function, and $\mu_{\text {old }}$ and $\sigma_{\text {old }}$ denote the distance between the distributions and the estimated standard deviations of the old item distribution, respectively. $s$ denotes the new-to-old variance ratio. $H$ and $F$ denote hit and false alarm rates, respectively, and $z$ denotes the inverse of the unit-normal distribution function (for details, see Macmillan \& Creelman, 1991).

2. To support an implicit criterion learning interpretation, only those subjects were included in Experiment 3 who reported being unaware of the manipulation in a postexperiment questionnaire. This questionnaire initially asked subjects whether or not they believed that there was anything generally odd about the feedback, and then asked about their perceived utility of feedback for each test block and each type of response ("old" or "new"). Any subject who indicated that he or she felt that the feedback was in any way odd or that it was differentially useful for old or new responses was excluded.

(Manuscript received November 7, 2006; revision accepted for publication September 24, 2007.) 